\title{
DISTRIBUCIÓN DE NANOFANERÓFITAS DE HOJAS SUCULENTAS EN DOS COMUNIDADES LEÑOSAS DEL CHACO SEMIÁRIDO SALINO
}

\author{
ISTRIBUTION OF SUCCULENT-LEAVES NANOPHANEROPHYTES IN TWO WOODY \\ PLANT COMMUNITIES OF THE SEMI-ARID SALINE CHACO
}

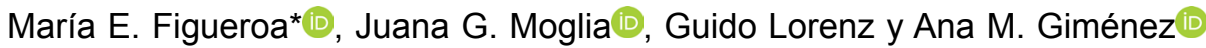

Instituto de Silvicultura y Manejo de Bosques, Facultad de Ciencias Forestales, Universidad Nacional de Santiago del Estero. Av. Belgrano (s) 1912, CP 4200, Santiago del Estero, Argentina

*meugeniaf83@gmail.com

Citar este artículo

FIGUEROA, M. E., J. G. MOGLIA, G. LORENZ \& A. M. GIMÉNEZ. 2019. Distribución de nanofanerófitas de hojas suculentas en dos comunidades leñosas del Chaco semiárido salino. Bol. Soc. Argent. Bot. 54: 277-294.

DOI: http://dx.doi. org/10.31055/1851.2372.v54. n2.24372

Recibido: 26 Octubre 2018 Aceptado: 29 Marzo 2019 Publicado: 30 Junio 2019 Editor: Guillermo Funes iD

\section{SUMMARY}

Background and aims: In the semi-arid saline Chaco, Argentina, the nanophanerophytes presence and the succulent leaf type are frequent. To determine if the occurrence of both traits is related to soil salinity, this paper describes the distribution of life forms and leaf types in relation to soil salinity between two woody plant communities.

M\&M: Sampling of woody plant and soil was carried out in both a scrubland and a xerophilous forest on 25 subplots of $100 \mathrm{~m}^{2}$ at each site. Soil electrical conductivity and $\mathrm{pH}$ were determined down to a depth of $50 \mathrm{~cm}$. Stratification and plant cover, abundance, species richness and diversity index were described. The degree of similarity between sites was evaluated according to the number of nanophanerophyte and succulent-leaves shared species, using the Sørensen coefficient. The species were grouped considering simultaneously their life form and leaf type. Relationships between salinity and plant groups were evaluated by means of redundancy analysis.

Results: Richness, diversity and structural complexity were greater in the forest. The similarity between sites was low. Succulent-leafed nanophanerophytes were dominant in the scrubland, with high salinity and $\mathrm{pH}$. In the forest, sclerophyllousleafed microphanerophytes were dominant, with low salinity and $\mathrm{pH}$.

Conclusion: The distribution of succulent-leaves nanophanerophytes presented differences according to the salinity of the soil of both woody communities.

\section{KEY WORDS}

Nanophanerophytes, salinity, semi-arid lands, succulent leaf, woody plants.

\section{RESUMEN}

Introducción y objetivos: En los hábitats salinos del Chaco semiárido es frecuente la presencia de la forma de vida nanofanerófita y el tipo de consistencia de hoja suculenta. Para determinar si la ocurrencia de ambos rasgos está relacionada a la salinidad del suelo, en este trabajo se describe la distribución de formas de vida y tipos de consistencia de hoja en relación a la salinidad del suelo entre dos comunidades leñosas.

M\&M: Se realizó un censo de leñosas y un muestreo de suelo en un matorral halófilo y en un bosque xerófilo sobre 25 subparcelas de $100 \mathrm{~m}^{2}$ en cada sitio. Se determinó $\mathrm{pH}$ y conductividad eléctrica del suelo hasta $50 \mathrm{~cm}$ de profundidad. Se describieron la cobertura y estratificación de la vegetación, riqueza, abundancia e índice de diversidad para especies leñosas. Se evaluó el grado de similitud entre sitios según la cantidad de especies de nanofanerófitas y de hoja suculenta compartidas, mediante el coeficiente de Sørensen. Las especies fueron agrupadas considerando simultáneamente su forma de vida y tipo de consistencia de hoja. La relación entre salinidad y grupos de leñosas se evaluó mediante un análisis de redundancia.

Resultados: La riqueza, diversidad y complejidad estructural fueron mayores en el bosque. La similitud entre sitios fue baja. Las nanofanerófitas de hojas suculentas dominaron en el matorral con elevada salinidad y $\mathrm{pH}$. Las microfanerófitas de hojas semi-coriáceas fueron dominantes en el bosque con baja salinidad y $\mathrm{pH}$.

Conclusión: La distribución de las nanofanerófitas de hojas suculentas presentó diferencias según la salinidad del suelo de ambas comunidades leñosas.

Palabras clave

Leñosa, nanofanerófita, salinidad, semiárido, suculenta. 


\section{INTRODUCCIÓN}

La salinización del suelo es frecuente en las regiones áridas y semiáridas. Los ambientes semiáridos salinos presentan una alta heterogeneidad ambiental dada por la variabilidad espacio temporal de los factores edáficos en general y de las sales solubles en particular (Schofield \& Kirkby, 2003; Yang et al., 2011; Bui, 2013; Panta et al., 2014). Existe un vasto conocimiento en cuanto a riqueza, diversidad, composición, endemismos, rasgos funcionales, cobertura, estructura y fisionomía de la vegetación de hábitats salinos semiáridos y su variación a lo largo de gradientes de salinidad (Braun-Blanquet, 1979; Schlesinger et al., 1996; Díaz \& Cabido, 1997; Wilder et al., 2008; Eggli \& Nyffeler, 2009; Grigore et al., 2010; Huchzermeyer \& Flowers, 2013; Atia et al., 2014). Numerosos estudios se han realizado en diferentes ecosistemas salinos áridos y semiáridos del planeta intentando comprender de qué manera la heterogeneidad ambiental influye en los patrones de distribución de la vegetación y los mecanismos por los cuales las especies logran establecerse (Larcher, 1977; Grigore et al., 2010; Piernik, 2003; Bui, 2013). El rol ecológico de cada una de estas especies dependerá en gran medida de sus características morfológicas más sobresalientes, como tamaño individual, arquitectura, forma de vida y densidad (Chapin, 1993; Bertiller et al., 2004).

En los distritos halofíticos del Chaco semiárido (Ragonese \& Castiglione, 1970) dos tipos de rasgos que se destacan entre las especies leñosas dominantes son la forma de vida y el tipo de consistencia de hoja. Dominan dos formas de vida: una especie de microfanerófita, Prosopis ruscifolia (Fabaceae) y nanofanerófitas suculentas de la familia Chenopodiaceae. Las especies están distribuidas en las siguientes unidades ambientales: del Salado, con Prosopis ruscifolia y Allenrolfea vaginata; Mar Chiquita, con Salicornia ambigua; Salinas Grandes, con Allenrolfea patagonica y Heterostachys ritteriana. Prosopis ruscifolia tolera concentraciones salinas equivalentes al agua de mar (Meloni et al., 2008) y presenta un rápido crecimiento (espesor medio de anillos de 5,66 $\mathrm{mm}$ ) comparado con otras especies del género y otras especies arbóreas principales de la región Chaqueña semiárida (Ríos et al., 2008; Giménez et al., 2009). Crece en suelos salinos asociados a llanuras de inundación y forma bosques bajos, puede presentar porte arbustivo o arbóreo según el tipo de hábitat (Giménez et al., 2009; Giménez \& Moglia, 2003). Allenrolfea vaginata es una especie de porte arbustivo de crecimiento bajo a medio (espesor medio de anillos de $3,85 \mathrm{~mm}$ ) y posee hojas suculentas (Giménez et al., 2008). Ambas especies presentan una alta capacidad de colonizar suelos afectados por sales solubles, ocupando extensas superficies dando lugar a 'vinalares' $(P$. ruscifolia) y 'jumeales' (A. vaginata), nombres que reciben localmente estas comunidades vegetales (Cabrera, 1976; Giménez \& Moglia, 2003; Astrada \& Adámoli, 2005).

Otro rasgo recurrente que comparten las especies que crecen en suelos extremadamente salinos es la hoja suculenta. Las halófitas de hojas suculentas poseen un rol ambiental destacado en la fitorremediación y desalinización de suelos afectados por sales y metales pesados, gracias al mecanismo de dilución de sales en las hojas (Türkan \& Demiral, 2009; Ruan et al., 2010; Panta et al., 2014; Nouri et al., 2017). La importancia de identificar patrones recurrentes entre los rasgos de las plantas dominantes en diferentes condiciones ambientales, radica en poder predecir la magnitud y tendencia de los procesos ecosistémicos (Díaz \& Cabido, 1997). En la vegetación halofítica del centro-oeste de Argentina, la predominancia de rasgos como hojas suculentas, alta ramificación, órganos protegidos por ceras y pelos, estaría relacionada a una baja producción de biomasa, lento ciclado de nutrientes, baja fijación de carbono y escasa retención de agua por la vegetación (Díaz \& Cabido, 1997).

Sin embargo, son escasos los estudios sobre la relación entre los rasgos de la vegetación leñosa, la heterogeneidad ambiental y los procesos ecológicos dentro de los hábitats salinos del Chaco semiárido. En estos hábitats, la alta recurrencia de la forma de vida nanofanerófita y el tipo de consistencia de hoja suculenta, permitiría agrupar a las especies que comparten ambos rasgos en un tipo funcional respuesta a condiciones de salinidad. Para analizar esta relación, en este trabajo se describe simultáneamente la distribución de formas de vida y tipos de consistencia de hoja en relación a variaciones locales de la salinidad del suelo entre dos comunidades leñosas de un hábitat salino semiárido. 


\section{Materiales y Métodos}

\section{Sitio de estudio}

El área de estudio se ubica sobre la unidad geomorfológica del valle fluvial del río Salado, en la antigua llanura aluvial del río Mailín, departamento Salavina, provincia de Santiago del Estero, Argentina.

El clima es semiárido, mesotermal con una temperatura media anual de $20,8{ }^{\circ} \mathrm{C}$. La precipitación media anual es de $650 \mathrm{~mm}$, la evapotranspiración media anual es de $1450 \mathrm{~mm}$, con un déficit climático medio anual de $800 \mathrm{~mm}$ (Prieto et al., 2006).

El río Mailín es un curso de agua estrecho que se formó por desbordes del río Salado, pero que en la actualidad no transporta agua (Subsecretaría de Recursos Hídricos de la Nación Argentina, 1979). Las poblaciones humanas locales utilizan algunos sectores del cauce como aguadas para los animales y huertos para el consumo familiar.

La vegetación corresponde al distrito halofítico del Salado del Chaco semiárido. En el cauce propiamente dicho la vegetación es abierta con escasa cobertura arbórea, algunos árboles emergentes y un estrato arbustivo bajo. A ambos lados del cauce existen albardones de relieve convexo originados por la acumulación de material fluvial, los cuales, al estar cubiertos por vegetación forman islas dentro de la llanura aluvial. La llanura propiamente dicha es el elemento del paisaje de mayor extensión superficial. Se extiende a ambos lados del cauce. Presenta una matriz de suelo desnudo con pequeños montículos (de menor dimensión y diferente origen que los albardones) sobre los cuales crecen matas de Allenrolfea vaginata, cactus arborescentes y algunos individuos aislados de especies arbóreas, principalmente Prosopis ruscifolia. La vegetación contigua a la llanura, hacia el este, es un bosque xerófito bajo y abierto de Schinopsis lorentzii y Aspidosperma quebracho blanco, y hacia el oeste, es un bosque de similares características pero que a diferencia del primero, se ubica en un relieve deprimido que recibe inundaciones en épocas de crecientes del río Dulce.

Este trabajo se realizó en dos de las comunidades leñosas descriptas asociadas a la influencia del río Mailín: el matorral de Allenrolfea vaginata de la antigua llanura aluvial, por ser de mayor extensión, y el bosque xerófilo contiguo a la llanura hacia al este, por estar ubicado sobre un relieve más estabilizado que no está sujeto a inundaciones. Las variaciones de relieve y mircorrelieve entre ambas comunidades pueden ser significativas tanto para la distribución de las sales en el suelo, como para la vegetación. Con el objetivo de describir la distribución de grupos de leñosas con rasgos morfológicos comunes y su relación con variaciones locales de la salinidad del suelo, se seleccionaron el matorral y el bosque por ser comunidades contiguas y con características contrastantes a pesar de pertenecer a la misma unidad ambiental.

El suelo es salino en ambos sitios ocupados por los dos tipos de vegetación. En el matorral, el suelo es un Fluvic Solonchak (Siltic) (IUSS Working Group WRB, 2014) / Aridic Ustifluvent (Soil Taxonomy, 2014). En el bosque, el suelo es un Haplic Solonchak (IUSS Working Group WRB, 2014) / Aridic Ustiorthent (Soil Taxonomy, 2014) (descripción realizada en estudios previos según las terminologías de IUSS Working Group WRB, 2014, Soil Taxonomy, 2014 y Moscatelli, 1990). Ambos sitios se ubican a una distancia aproximada

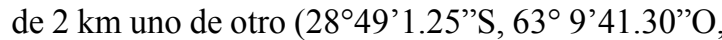
Fig. 1A; $28^{\circ} 48^{\prime} 14.76^{\prime \prime}$, $63^{\circ}$ 9'5.04”'O, Fig. 1B).

\section{Muestreo y análisis de datos}

En cada comunidad se muestreó el suelo y la vegetación leñosa en un área de $50 \mathrm{~m} \times 50 \mathrm{~m}$, que fue subdividida en 25 parcelas de $10 \mathrm{~m} \times 10 \mathrm{~m}$. En cada parcela se contaron los individuos de las especies leñosas (arbustos y árboles) mayores de 20 $\mathrm{cm}$ de altura.

El muestreo de suelo se realizó en cada parcela extrayendo tres muestras al azar. Las muestras se tomaron en dos profundidades: 0 a $20 \mathrm{~cm}$ y 20 a $50 \mathrm{~cm}$. Para ello se emplearon dos barrenos de $1,4 \mathrm{~cm}$ de diámetro. Las tres muestras tomadas al azar en cada parcela conformaron una misma muestra compuesta por parcela y profundidad. Las muestras se llevaron al laboratorio, se secaron al aire y se tamizaron a través de una malla de 2 $\mathrm{mm}$. Se prepararon suspensiones de proporción de proporción suelo / agua de 1:2,5, $15 \mathrm{~mL}$ de agua destilada a $6 \mathrm{~g}$ de suelo. Como parámetros del suelo se determinaron la salinidad, mediante la conductividad eléctrica $(\mathrm{CE}, \mathrm{dS} / \mathrm{m})$, y el $\mathrm{pH}$, en un extracto 1:2,5. La denominación de ambos parámetros fue la siguiente, según la profundidad 

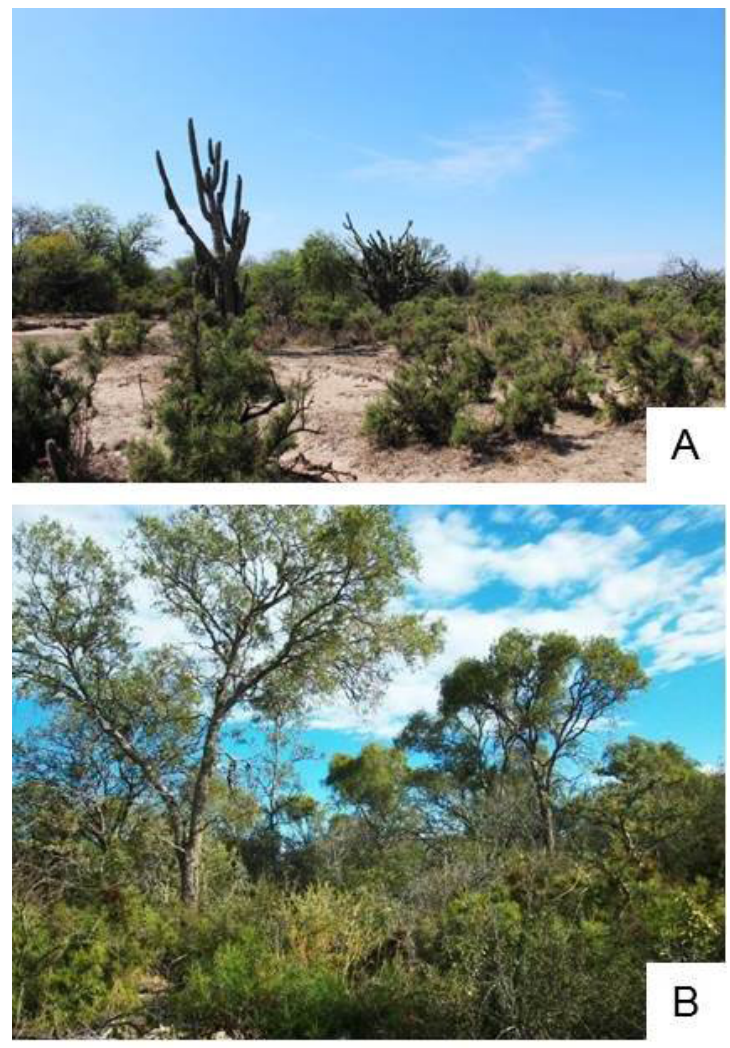

Fig. 1. Comunidades leñosas estudiadas. A: Matorral halófilo de Allenrolfea vaginata y Prosopis ruscifolia. B: Bosque bajo con influencia salina de Schinopsis lorentzii y Aspidosperma quebracho blanco.

analizada: CE20 y pH20, de 0-20 cm, CE50 y pH50, de $20-50 \mathrm{~cm}$. A partir de los valores de CE del extracto, se estimó la CE en capacidad de campo, siguiendo la transformación propuesta por FAO (2006) para permitir así la interpretación genética y ecológica del tenor de salinidad. Los análisis fueron realizados en el laboratorio de suelos del Instituto de Silvicultura y Manejo de Bosques de la Facultad de Ciencias Forestales de la Universidad Nacional de Santiago del Estero. Para caracterizar el suelo de cada sitio según $\mathrm{CE}$ y $\mathrm{pH}$, los valores de las variables edáficas $\mathrm{CE}$ y $\mathrm{pH}$ de cada parcela se ponderaron según la profundidad: otorgando un valor de 2 puntos para la profundidad $0-20 \mathrm{~cm}$ y 3 puntos para la profundidad $20-50 \mathrm{~cm}$. Luego, con los valores ponderados, se calculó el promedio general para cada variable por sitio. La ponderación se realizó mediante la siguiente ecuación:

$$
\mathrm{Ve}=((\mathrm{Vi} \times 2)+(\mathrm{Vj} \times 3)) / 5
$$

Dónde:

$V e=$ valor de la variable edáfica $(\mathrm{CE} ; \mathrm{pH})$ ponderado por la profundidad

$V i=$ valor de la variable edáfica $(\mathrm{CE} ; \mathrm{pH})$ medido en la profundidad $0-20 \mathrm{~cm}$

$V j=$ valor de la variable edáfica $(\mathrm{CE} ; \mathrm{pH})$ medido en la profundidad $20-50 \mathrm{~cm}$

Las variables $\mathrm{CE}, \mathrm{pH}$ y sus valores medios ponderados se compararon estadísticamente entre sitios mediante una prueba $\mathrm{T}$ para muestras independientes, calculada con el programa Infostat (Di Rienzo et al., 2014).

Para caracterizar la vegetación del matorral y del bosque se realizó una estimación visual de la cobertura según los grados y porcentajes de cobertura de la clasificación de Margalef (1974); de la estratificación horizontal considerando las clases cerrada, abierta, dispersa; y de la estratificación vertical según los estratos arbóreo, arbustivo, herbáceo y de cactáceas, para este último se registró la presencia de especies de la familia Cactaceae. La nomenclatura se consultó en las páginas en línea de Flora Argentina (2017) y The Plant List (2013).

Con base en el relevamiento de especies se caracterizó la riqueza y diversidad de leñosas de cada sitio. Se determinó la abundancia, riqueza e índice de diversidad Shannon- Wiener $\left(H^{\prime}\right)$ por parcela y por sitio. Los cálculos se realizaron con las funciones 'specnumber' y 'diversity' del paquete estadístico 'vegan v. 2.4-6' (Oksanen et al., 2018) en el entorno R (R CoreTeam, 2015). Para cada variable se calculó el valor global o total para el sitio y el promedio y desvío estándar (sd), considerando las 25 parcelas por sitio. Se incluyó una prueba $\mathrm{T}$ entre sitios para cada variable.

Las especies se clasificaron según la consistencia de la hoja y la forma de vida. Las categorías consideradas para el rasgo consistencia de hoja fueron: suculenta o carnosa, coriácea y semi-coriácea (Vendramini et al., 2002). Las categorías para el atributo forma de vida se seleccionaron teniendo en cuenta la clasificación de Ragonese (1951): i) caméfita (arbustos $<0,3 \mathrm{~m}$ de altura); ii) nanofanerófita (leñosa de 0,3 a $2 \mathrm{~m}$ ); iii) microfanerófita (leñosa de 2 a 8 m) y iv) mesofanerófita (árboles de 8 a 12 m). 


\section{E. Figueroa et al. - Nanofanerófitas suculentas y salinidad}

La asignación de rasgos a las especies se realizó a partir de observaciones en campo, referencias bibliográficas y descripciones botánicas (Ragonese, 1951; Miller, 1989; Cavanna et al., 2010; Arambarri et al., 2011; Arambarri et al., 2012; Pérez Cuadra \& Cambi, 2014; Flora Argentina, 2017).

Teniendo en cuenta que la forma de vida nanofanerófita y la hoja suculenta son los rasgos más recurrentes en los hábitats salinos, se evaluó el grado de similitud entre ambas comunidades en cuanto a dichos rasgos. Para ello, se adaptó el cálculo del coeficiente de similitud de Sørensen cuantitativo (IScuant) para ambos rasgos por separado (Moreno, 2001; Magurran, 2004). Este coeficiente expresa el grado en el que dos muestras son semejantes por las especies presentes en ellas (Moreno, 2001). El calculó se realizó con base en la composición florística global y en las abundancias por especie de cada comunidad leñosa, considerando el número total de individuos contabilizados en cada sitio y la abundancia por especie de hoja suculenta (IScuant ${ }_{S}$ )

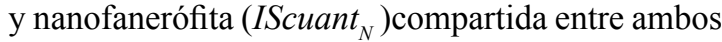
sitios, según la siguiente ecuación:

$$
\text { IScuant }=2 \mathrm{pN} /(\mathrm{aN}+\mathrm{bN})
$$

Dónde:

$a N=$ número total de individuos en el sitio A

$b N=$ número total de individuos en el sitio $\mathrm{B}$

$p N=$ suma de la menor de las dos abundancia para las especies de hojas suculentas / nanofanerófitas encontradas en ambos sitios

Existe una mayor similitud entre sitios cuando el valor del índice es cercano a 1 (Moreno, 2001).

Las especies leñosas relevadas en ambos sitios se agruparon según rasgos comunes. Para ello se construyó una tabla de doble entrada con las categorías de forma de vida y tipo de consistencia de hoja. En cada combinación de categorías se colocó el número de especies presentes, a las cuales luego se le asignó un número de grupo. La abundancia absoluta de cada grupo dentro de una parcela fue la suma de los individuos de las especies que conforman un grupo. La abundancia relativa fue la proporción de este valor respecto al número total de individuos de cada parcela. Para evaluar si la abundancia de los grupos de leñosas difiere entre ambos sitios o comunidades leñosas se realizó un PERMANOVA (Análisis permutacional multivariado de varianza) empleando la función 'adonis' del paquete estadístico 'vegan' considerando la matriz de abundancias relativas de cada grupo de leñosas por parcela en función de cada sitio.

La relación entre los grupos definidos y las variables edáficas se evaluó mediante un análisis de redundancia (RDA). El RDA se realizó con la función 'rda' del paquete 'vegan' antes citado. El análisis se realizó con dos matrices primarias: a) matriz de abundancia de leñosas por grupo, parcela y sitio; b) matriz de variables edáficas con los valores de CE20, pH20, CE50 y pH50, por parcela y sitio. Las variables se estandarizaron siguiendo las recomendaciones de Oksanen et al. (2018), usando la matriz de correlaciones, en lugar de la matriz de varianzas, para evitar que las variables con alta varianza afecten a las menos variables y obtener una ordenación balanceada. La salida del RDA se representó en un gráfico biplot. La significancia del RDA se evaluó a través de un test de permutaciones (Oksanen et al., 2018).

\section{Resultados}

El suelo del matorral presentó mayor salinidad y alcalinidad que el suelo del bosque, en el perfil analizado $(0$ a $50 \mathrm{~cm}$ ), según los valores de $\mathrm{CE}$ y $\mathrm{pH}$ ponderados (Tabla 1). Esta condición del suelo sugiere un hábitat más estresante en el matorral. En la figura 2 se muestran los valores de CE obtenidos por parcela, profundidad y tipo de comunidad vegetal. Los valores de CE dentro de cada sitio mostraron una alta variabilidad entre las parcelas en ambas profundidades (alto coeficiente de variación). En ambos sitios la CE entre los 20 y $50 \mathrm{~cm}$ de profundidad fue aproximadamente el doble que la registrada en los primeros $20 \mathrm{~cm}$.

La cobertura vegetal en la parcela del matorral fue entre el 25 y $30 \%$, grado 3 en la escala de Margalef (1974). La estructura horizontal fue dispersa, con parches vegetados intercalados con suelo desprovisto de vegetación vascular. Se identificaron tres estratos vegetales: i) inferior, de gramíneas y crasas; ii) arbustivo, de $<2,5$ metros de altura; iii) estrato de cactáceas. Entre las especies de Cactaceae se identificaron las siguientes: Echinopsis rhodotricha ('alao'), Echinopsis leucantha ('sitki'), Gymnocalycium schickendantzii ('miyoga'), Cleistocactus baumannii ('ullivincha'), Opuntia quimilo ('quimil') y Stetsonia coryne ('cardón'). 
Tabla 1. Valores de CE (dS/m) y pH del suelo del matorral y del bosque. Se presenta valor de: Media aritmética \pm desvío estándar (sd) (mínimo-máximo) CV (coeficiente de variación). CEp: valor promedio de la conductividad eléctrica ponderada por la profundidad; pHp: valor promedio del pH ponderado por

la profundidad. *Diferencia no significativa según prueba T para muestras independientes, $p$-valor $>$

$0,05 .{ }^{* *}$ Diferencia significativa según prueba T para muestras independientes, $p$-valor $=0,0001$, nivel de significación $\alpha=0,05$. CE en (dS/m).

\begin{tabular}{|c|c|c|}
\hline Suelo & Matorral halófilo & Bosque xerófilo \\
\hline CE20 & $12,83 \pm 11,02(2,44-52,05) C V=85,94$ * & $10,22 \pm 6,51(2,37-23,91) C V=63,71$ * \\
\hline CE50 & $36,89 \pm 12,04(14,23-57,04) \mathrm{CV}=32,64$ ** & $21,5 \pm 10,75(3,09-42,28) C V=50,05$ ** \\
\hline CEp & $27,27 \pm 9,37(10,03-46,47) C V=34,39 * *$ & $16,98 \pm 8,87(3,05-34,93) C V=52,23$ ** \\
\hline $\mathrm{pH} 20$ & $9,62 \pm 0,45(8,5-10,34) C V=4,7$ ** & $7,57 \pm 0,83(6,48-9,45) C V=10,20$ ** \\
\hline $\mathrm{pH} 50$ & $9,25 \pm 0,62(8,23-10,27) C V=6,69 * *$ & $7,84 \pm 0,63(6,55-8,92) C V=8,04$ ** \\
\hline $\mathrm{pHp}$ & $9,4 \pm 0,47(8,66-10,22) C V=5,1 * *$ & $7,73 \pm 0,68(6,52-9,04) C V=8,88$ ** \\
\hline
\end{tabular}
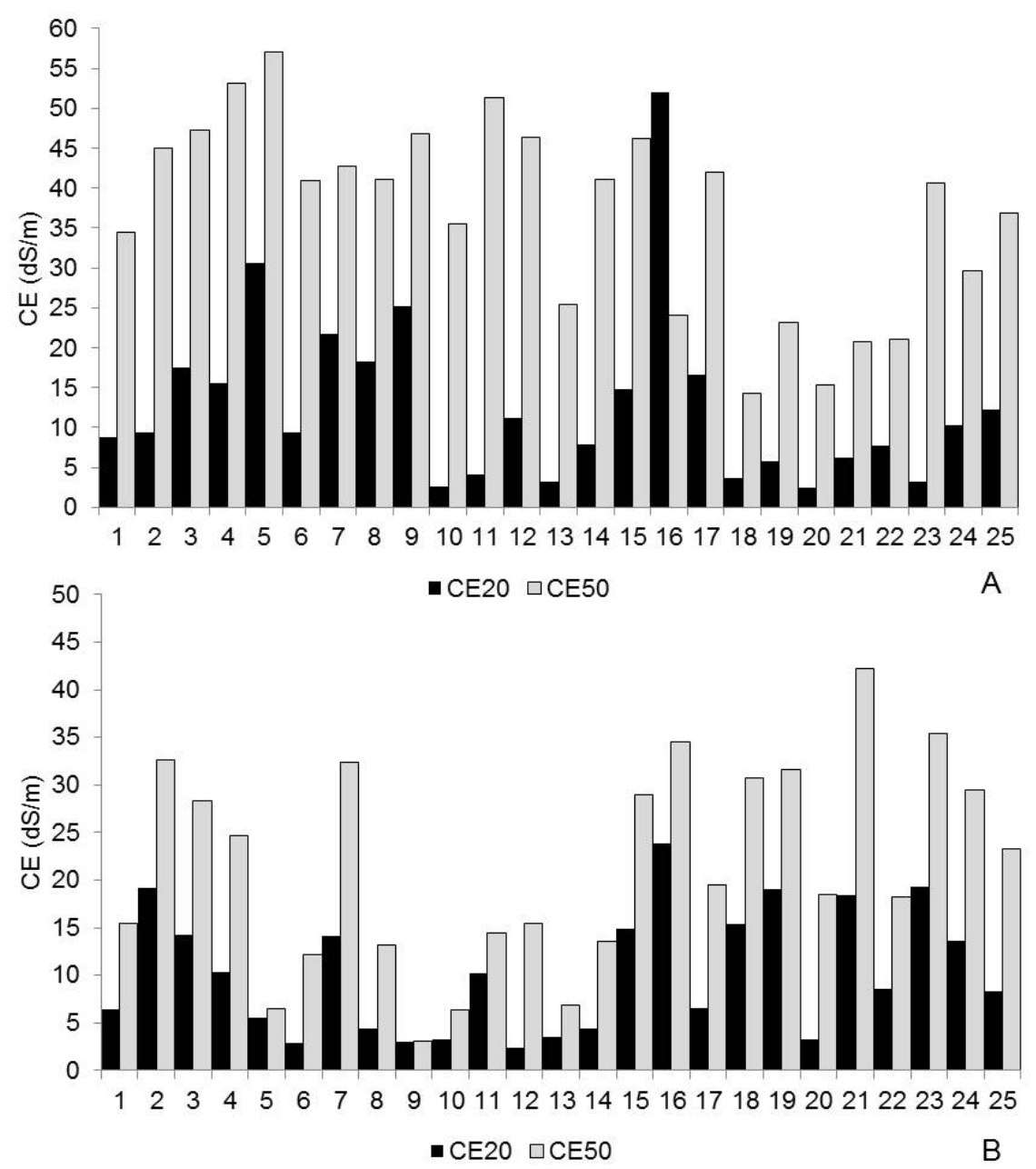

Fig. 2. Valores de conductividad eléctrica ( $\mathrm{dS} / \mathrm{m}$ ) medidos en dos profundidades, CE20 y CE50, en el suelo del matorral (A) y del bosque (B). Umbrales críticos de salinidad de $8 \mathrm{dS} / \mathrm{m}$ (con pH $\geq 8.5$ ) y $15 \mathrm{dS} / \mathrm{m}$ (FAO, 2006). 


\section{E. Figueroa et al. - Nanofanerófitas suculentas y salinidad}

El bosque presentó una cobertura vegetal entre el 50 y $75 \%$, grado 4 de la escala de Margalef (1974). La estratificación horizontal fue abierta. Los estratos verticales se clasificaron en: i) estrato arbóreo, con ejemplares emergentes aislados de Schinopsis lorentzii y Aspidosperma quebrachoblanco, cuya altura media fue de 10 metros; ii) estrato arbóreo secundario, con Prosopis nigra, Ziziphus mistol y Senegalia praecox; iii) estrato arbustivo, con Celtis pallida, Atamisquea emarginata y Maytenus vitis-idaea; iv) estrato de cactáceas, con Monvillea spegazzinii, Opuntia anacantha Speg. var. retrorsa, Cleistocactus baumannii, Harrisia pomanensis, Cereus forbesii y Stetsonia coryne.

Las leñosas relevadas en total fueron 31 especies entre ambos sitios, 18 en el matorral y 28 en el bosque (Tabla 2). En las figuras 3 y 4 se muestran imágenes de las especies con hojas suculentas. El índice de diversidad $H^{\prime}$ promedio y la riqueza media fueron significativamente mayores en el bosque que en el matorral (Tabla 2). La densidad de especies en el matorral fue de alrededor de 7 especies por $100 \mathrm{~m}^{2}$; en tanto que en el bosque la densidad de especies fue de 10 especies por $100 \mathrm{~m}^{2}$. En el matorral predominaron, sobre el total de individuos contabilizados, los individuos leñosos con hojas suculentas (84\%), seguidos por los de hojas semi-coriáceas (9\%) y coriáceas $(7 \%)$. En el bosque la distribución de tipos de hojas fue más equitativa: $41 \%$ hojas semi-coriáceas, $32 \%$ hojas suculentas y $26 \%$ hojas coriáceas. En cuanto a las formas de vida, en el matorral predominaron las nanofanerófitas con el $92 \%$ de los individuos contabilizados, sobre las demás formas de vida, caméfitas $(7 \%)$, microfanerófitas $(1 \%)$ y mesofanerófitas $(0,1$ $\%)$. En el bosque los individuos nanofanerófitos fueron también los más abundantes (53\% de los individuos leñosos contabilizados), seguidos por los microfanerófitos (42\%) y escasamente representados los mesofanerófitos (3\%) y caméfitos $(1 \%)$.

El grado de similitud entre el bosque y el matorral fue bajo (menor a 1) según el coeficiente de Sørensen calculado para las especies de hojas suculentas (IScuant $\mathrm{s}=0,23$ ) y nanofanerófitas $\left(\right.$ IScuant $\left._{\mathrm{N}}=0,24\right)$. Es decir que ambos sitios poseen un bajo número de especies de hojas suculentas y nanofanerófitas en común.
La asociación entre los caracteres forma de vida y consistencia de hoja fue significativa según el análisis de la tabla de contingencia $(\mathrm{p}=0,01)$ lo que permitió clasificar a las 31 especies relevadas en seis grupos (Tabla 3) considerando simultáneamente ambos rasgos: G1 (9 especies) microfanerófitas semi-coriáceas, G2 (3 especies) microfanerófitas coriáceas, G3 (9 especies) nanofanerófitas suculentas, G4 (3 especies) nanofanerófitas semicoriáceas, G5 (2 especies) caméfitas suculentas, G6 (5 especies) especies de hoja coriácea y semicoriácea de formas de vida varias.

En las Tablas 4 y 5 se muestran la abundancia relativa y absoluta de cada grupo de leñosas por parcela en el matorral y en el bosque respectivamente. Los grupos más representativos fueron el G1, G3 y G6. El grupo G1 de las microfanerófitas semi-coriáceas y el G6 de leñosas de hojas coriáceas y semicoriáceas presentaron una abundancia más alta en el bosque; en tanto que el grupo G3 de nanofanerófitas suculentas fue más abundante en el matorral. La abundancia relativa de los grupos de leñosas fue significativamente diferente entre ambas comunidades, según el resultado del PERMANOVA $(p=0,01)$.

El RDA explicó el $25 \%$ de la variancia total (Fig. 5). El RDA1 fue el único eje significativo, $\mathrm{p}=0,001$, y explicó el $22 \%$ de la variabilidad total. Las variables edáficas que mejor explicaron la distribución de los grupos de especies fueron la CE50, $p=0,001$ y el $p H 20, p=0,001$. Las nanofanerófitas de hojas suculentas (G3), las de hojas semi-coriáceas (G4) y las caméfitas suculentas (G5) estuvieron asociadas a valores elevados de salinidad y $\mathrm{pH}$. De manera opuesta, las microfanerófitas semi-coriáceas (G1), las especies del grupo G6 y G2 estuvieron asociadas a valores bajos de salinidad y $\mathrm{pH}$. Los grupos G1 y G3 tuvieron mayor correlación con el RDA1.

El eje RDA1 sugiere un gradiente de salinidad marcado por el pH20 y la $\mathrm{CE}$ entre los 20 y 50 $\mathrm{cm}$ de profundidad. En dicha profundidad la $\mathrm{CE}$ tuvo los valores más elevados de todo el perfil en ambos sitios. Respecto al mismo eje, el grupo G3 presentó la mayor correlación positiva y el grupo G1 la mayor correlación negativa. Es decir, los grupos G3 y G1 se ubicaron en los extremos del gradiente de salinidad. Los cambios en la abundancia relativa de ambos grupos indican 
Tabla 2. Listado de especies leñosas relevadas por sitio. Riqueza (S), abundancia e índice de diversidad $\left(H^{\prime}\right)$. Densidad de individuos expresada en número de individuos por hectárea; sd (desvío estándar). * Diferencias significativas según prueba T para muestras independientes, nivel de significación $\alpha=0,05$.

\begin{tabular}{|c|c|c|c|c|c|}
\hline \multirow[b]{2}{*}{ Familia } & \multirow[b]{2}{*}{ Especie } & \multirow{2}{*}{$\begin{array}{l}\text { Consistencia } \\
\text { de hoja }\end{array}$} & \multirow[b]{2}{*}{ Forma de vida } & \multicolumn{2}{|c|}{ Densidad de } \\
\hline & & & & $\begin{array}{l}\text { Matorral } \\
\text { halófito }\end{array}$ & $\begin{array}{l}\text { Bosque } \\
\text { xerófito }\end{array}$ \\
\hline AMARANTHACEAE & Alternanthera nodifera & Suculenta & Caméfita & 20 & 0 \\
\hline ANACAMPSEROTACEAE & Grahamia bracteata & Suculenta & Nanofanerófita & 564 & 264 \\
\hline ANACARDIACEAE & Schinopsis lorentzii & Semi-coriácea & Mesofanerófita & 0 & 40 \\
\hline ANACARDIACEAE & Schinus bumelioides & Semi-coriácea & Microfanerófita & 0 & 4 \\
\hline APOCYNACEAE & $\begin{array}{c}\text { Aspidosperma } \\
\text { quebracho- blanco }\end{array}$ & Coriácea & Mesofanerófita & 4 & 112 \\
\hline APOCYNACEAE & Vallesia glabra & Semi-coriácea & Microfanerófita & 0 & 4 \\
\hline ASTERACEAE & Cyclolepis genistoides & Suculenta & Nanofanerófita & 8 & 20 \\
\hline BORAGINACEAE & Ehretia cortesia & Suculenta & Caméfita & 0 & 24 \\
\hline CAPPARACEAE & Atamisquea emarginata & Coriácea & Nanofanerófita & 16 & 956 \\
\hline CELASTRACEAE & Maytenus vitis-idaea & Suculenta & Nanofanerófita & 0 & 152 \\
\hline CELASTRACEAE & Maytenus spinosa & Coriácea & Microfanerófita & 0 & 20 \\
\hline CELTIDACEAE & Celtis pallida & Semi-coriácea & Microfanerófita & 8 & 1036 \\
\hline CHENOPODIACEAE & Allenrolfea vaginata & Suculenta & Nanofanerófita & 2476 & 516 \\
\hline FABACEAE & Prosopis sericantha & Semi-coriácea & Nanofanerófita & 72 & 32 \\
\hline FABACEAE & Prosopis reptans & Semi-coriácea & Nanofanerófita & 512 & 0 \\
\hline FABACEAE & Prosopis vinalillo & Coriácea & Microfanerófita & 16 & 20 \\
\hline FABACEAE & Prosopis ruscifolia & Coriácea & Microfanerófita & 28 & 80 \\
\hline FABACEAE & Prosopis nigra & Semi-coriácea & Microfanerófita & 12 & 44 \\
\hline FABACEAE & Parkinsonia praecox & Semi-coriácea & Microfanerófita & 4 & 36 \\
\hline FABACEAE & Geoffroea decorticans & Semi-coriácea & Microfanerófita & 0 & 20 \\
\hline FABACEAE & Mimosa detinens & Semi-coriácea & Microfanerófita & 0 & 244 \\
\hline FABACEAE & Senegalia praecox & Semi-coriácea & Microfanerófita & 0 & 448 \\
\hline OLACACEAE & Ximenia americana & Semi-coriácea & Nanofanerófita & 0 & 4 \\
\hline RHAMNACEAE & Sarcomphalus mistol & Semi-coriácea & Microfanerófita & 0 & 80 \\
\hline RHAMNACEAE & Condalia microphylla & Coriácea & Nanofanerófita & 0 & 28 \\
\hline SOLANACEAE & Lycium athium & Suculenta & Nanofanerófita & 1648 & 400 \\
\hline SOLANACEAE & $\begin{array}{l}\text { Lycium tenuispinosum } \\
\text { var. friesii }\end{array}$ & Suculenta & Nanofanerófita & 592 & 0 \\
\hline SOLANACEAE & Lycium americanum & Suculenta & Nanofanerófita & 264 & 56 \\
\hline SOLANACEAE & Lycium ciliatum & Suculenta & Nanofanerófita & 180 & 124 \\
\hline SOLANACEAE & $\begin{array}{c}\text { Lycium } \\
\text { boerhaviaefolium }\end{array}$ & Suculenta & Nanofanerófita & 0 & 8 \\
\hline VERBENACEAE & Lippia salsa & Coriácea & Caméfita & 432 & 48 \\
\hline S (Riqueza de especies) & & & & 18 & 28 \\
\hline S promedio por parcela (10 & & & & 6,96 * & 10,64 * \\
\hline sd S & & & & 1,81 & 2,98 \\
\hline $\mathrm{N}^{\circ}$ Total de individuos & & & & 1714 & 1205 \\
\hline Abundancia promedio por & cela & & & 68,56 * & 48,2 * \\
\hline sd abundancia & & & & 36,28 & 9,51 \\
\hline$H^{\prime}$ (Shannon- Wiener) & & & & 1,86 & 2,48 \\
\hline$H^{\prime}$ Promedio (Shannon- Wi & er) por parcela & & & 1,47 * & 1,93 * \\
\hline sd $H^{\prime}$ & & & & 0,22 & 0,32 \\
\hline
\end{tabular}



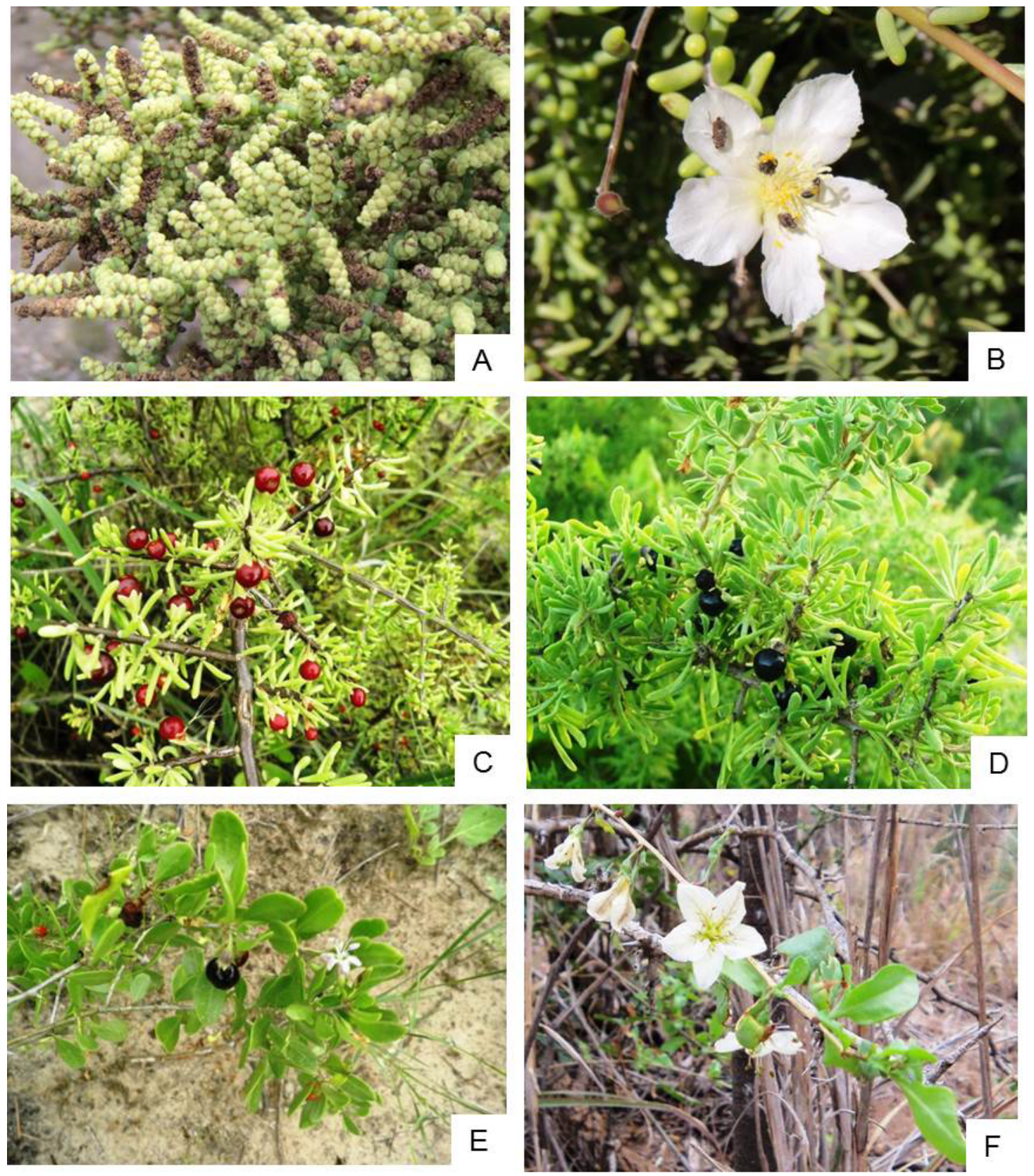

Fig. 3. Especies arbustivas leñosas de hojas suculentas relevadas en los muestreos del matorral y bosque. A. Allenrolfea vaginata. B. Grahamia bracteata. C. Lycium athium. D. Lycium tenuispinosum. E. Lycium americanum. F. Lycium ciliatum.

cambios en las condiciones de salinidad edáfica.

En la figura 6 se esquematizan los principales resultados encontrados. Los tenores de salinidad del suelo de ambos sitios fueron elevados y mostraron un aumento en profundidad. El valor de CE ponderado por la profundidad fue mayor en el matorral. La expresión de la vegetación fue diferente en ambas comunidades, con notables diferencias en la complejidad estructural, la riqueza y diversidad de especies leñosas. La 

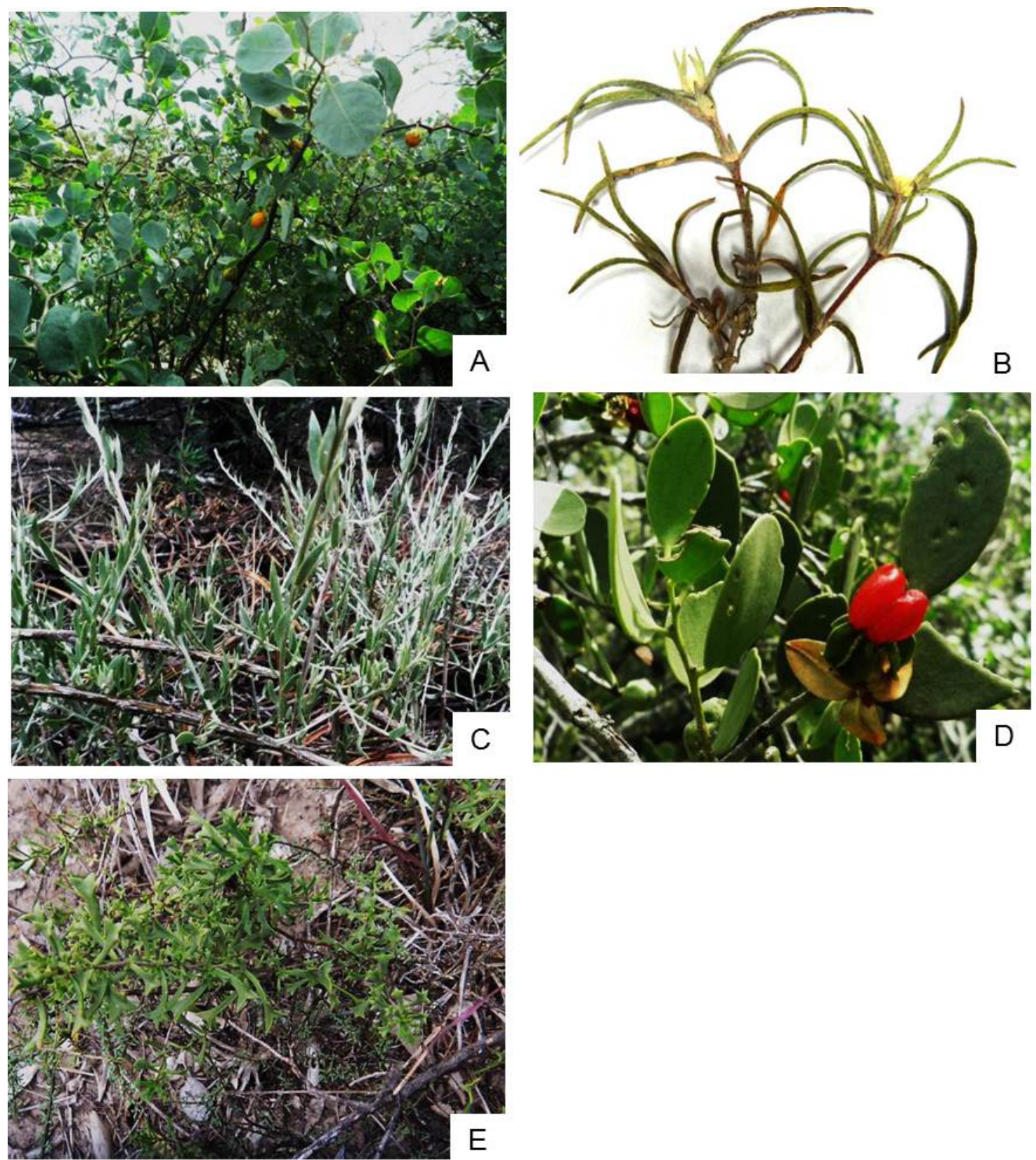

Fig. 4. A. Lycium boerhaviaefolium. B. Alternanthera nodifera. C. Cyclolepis genistoides. D. Maytenus vitisidaea. E. Ehretia cortesia.

comparación de la cantidad de especies de hojas suculentas y nanofanerófitas entre comunidades resultó en un bajo número de especies comunes o compartidas. La descripción porcentual de la abundancia de individuos por cada categoría de rasgo a nivel de comunidad, indicó una marcada predominancia de ciertas categorías en el matorral, en tanto que en el bosque la distribución fue más equitativa. La clasificación de las especies, considerando simultáneamente ambos rasgos, permitió identificar seis grupos de leñosas. La distribución de las abundancias de los 
Tabla 3. Tabla de contingencia para los rasgos forma de vida y consistencia de hoja. Entre paréntesis número de grupo asignado a las especies con rasgos comunes.

\begin{tabular}{|ccccc|}
\hline \multirow{2}{*}{ Forma de vida } & \multicolumn{4}{c|}{ Consistencia de hoja } \\
& Coriácea & Semi-corciácea & Suculenta & Total \\
\hline Caméfita & $1(\mathrm{G} 6)$ & 0 & $2(\mathrm{G} 5)$ & 3 \\
\hline Mesofanerófita & $1(\mathrm{G} 6)$ & $1(\mathrm{G} 6)$ & 0 & 2 \\
\hline Nanofanerófita & $2(\mathrm{G} 6)$ & $3(\mathrm{G} 4)$ & $9(\mathrm{G} 3)$ & 14 \\
\hline Microfanerófita & $3(\mathrm{G} 2)$ & $9(\mathrm{G} 1)$ & 0 & 12 \\
\hline Total & $\mathbf{7}$ & $\mathbf{1 3}$ & $\mathbf{1 1}$ & $\mathbf{3 1}$ \\
\hline
\end{tabular}

Tabla 4. Abundancia absoluta y relativa de grupos de leñosas por parcela en el matorral halófito. Total de individuos por grupo; abundancia relativa en valores decimales; sd: desvio estándar.

\begin{tabular}{|c|c|c|c|c|c|c|c|c|c|c|c|c|c|}
\hline \multirow{3}{*}{$\begin{array}{c}\text { Parcela } \\
1\end{array}$} & \multicolumn{12}{|c|}{ Grupos de leñosas por parcela en el matorral halófito } & \multirow{3}{*}{$\begin{array}{r}\text { Total } \\
110\end{array}$} \\
\hline & \multicolumn{2}{|c|}{ G1 } & \multicolumn{2}{|c|}{ G2 } & \multicolumn{2}{|c|}{ G3 } & \multicolumn{2}{|c|}{ G4 } & \multicolumn{2}{|c|}{ G5 } & \multicolumn{2}{|c|}{ G6 } & \\
\hline & 0 & 0 & 0 & 0 & 91 & 0,827 & 11 & 0,1 & 2 & 0,018 & 6 & 0,055 & \\
\hline 2 & 0 & 0 & 0 & 0 & 80 & 0,899 & 0 & 0 & 1 & 0,011 & 8 & 0,090 & 89 \\
\hline 3 & 0 & 0 & 0 & 0 & 79 & 0,814 & 4 & 0,041 & 0 & 0 & 14 & 0,144 & 97 \\
\hline 4 & 0 & 0 & 0 & 0 & 140 & 0,791 & 11 & 0,062 & 0 & 0 & 26 & 0,147 & 177 \\
\hline 5 & 0 & 0 & 0 & 0 & 78 & 0,876 & 5 & 0,056 & 0 & 0 & 6 & 0,067 & 89 \\
\hline 6 & 0 & 0 & 0 & 0 & 65 & 0,855 & 2 & 0,026 & 1 & 0,013 & 8 & 0,105 & 76 \\
\hline 7 & 0 & 0 & 0 & 0 & 100 & 0,735 & 27 & 0,199 & 1 & 0,007 & 8 & 0,059 & 136 \\
\hline 8 & 0 & 0 & 0 & 0 & 52 & 0,754 & 16 & 0,232 & 0 & 0 & 1 & 0,014 & 69 \\
\hline 9 & 0 & 0 & 0 & 0 & 61 & 0,968 & 0 & 0 & 0 & 0 & 2 & 0,032 & 63 \\
\hline 10 & 0 & 0 & 0 & 0 & 33 & 1 & 0 & 0 & 0 & 0 & 0 & 0 & 33 \\
\hline 11 & 0 & 0 & 0 & 0 & 20 & 0,833 & 1 & 0,042 & 0 & 0 & 3 & 0,125 & 24 \\
\hline 12 & 0 & 0 & 0 & 0 & 39 & 0,830 & 8 & 0,170 & 0 & 0 & 0 & 0 & 47 \\
\hline 13 & 0 & 0 & 1 & 0,018 & 42 & 0,764 & 12 & 0,218 & 0 & 0 & 0 & 0 & 55 \\
\hline 14 & 2 & 0,034 & 2 & 0,034 & 23 & 0,397 & 30 & 0,517 & 0 & 0 & 1 & 0,017 & 58 \\
\hline 15 & 0 & 0 & 0 & 0 & 61 & 1 & 0 & 0 & 0 & 0 & 0 & 0 & 61 \\
\hline 16 & 1 & 0,01 & 1 & 0,01 & 97 & 0,97 & 1 & 0,01 & 0 & 0 & 0 & 0 & 100 \\
\hline 17 & 0 & 0 & 0 & 0 & 43 & 0,956 & 0 & 0 & 0 & 0 & 2 & 0,044 & 45 \\
\hline 18 & 0 & 0 & 0 & 0 & 32 & 0,727 & 3 & 0,068 & 0 & 0 & 9 & 0,205 & 44 \\
\hline 19 & 0 & 0 & 1 & 0,034 & 18 & 0,621 & 7 & 0,241 & 0 & 0 & 3 & 0,103 & 29 \\
\hline 20 & 0 & 0 & 0 & 0 & 58 & 0,892 & 2 & 0,031 & 0 & 0 & 5 & 0,077 & 65 \\
\hline 21 & 0 & 0 & 1 & 0,021 & 45 & 0,957 & 0 & 0 & 0 & 0 & 1 & 0,021 & 47 \\
\hline 22 & 2 & 0,182 & 0 & 0 & 9 & 0,818 & 0 & 0 & 0 & 0 & 0 & 0 & 11 \\
\hline 23 & 0 & 0 & 1 & 0,018 & 43 & 0,768 & 4 & 0,071 & 0 & 0 & 8 & 0,143 & 56 \\
\hline 24 & 0 & 0 & 2 & 0,027 & 70 & 0,946 & 0 & 0 & 0 & 0 & 2 & 0,027 & 74 \\
\hline 25 & 1 & 0,017 & 2 & 0,034 & 54 & 0,915 & 2 & 0,034 & 0 & 0 & 0 & 0 & 59 \\
\hline Total & 6 & 0,243 & 11 & 0,197 & 1433 & 20,914 & 146 & 2,119 & 5 & 0,050 & 113 & 1,476 & 1714 \\
\hline Promedio & 0,24 & 0,010 & 0,44 & 0,008 & 57,32 & 0,837 & 5,84 & 0,085 & 0,2 & 0,002 & 4,52 & 0,059 & \\
\hline sd & 0,597 & 0,037 & 0,712 & 0,013 & 30,195 & 0,133 & 8,194 & 0,121 & 0,5 & 0,005 & 5,875 & 0,059 & \\
\hline
\end{tabular}


Tabla 5. Abundancia absoluta y relativa de grupos de leñosas por parcela en el bosque xerófito. Total de individuos por grupo; abundancia relativa en valores decimales; sd: desvío estándar.

\begin{tabular}{|c|c|c|c|c|c|c|c|c|c|c|c|c|c|}
\hline \multirow{3}{*}{$\begin{array}{c}\text { Parcela } \\
1\end{array}$} & \multicolumn{12}{|c|}{ Grupos de leñosas por parcela en el bosque xerófito } & \multirow{3}{*}{$\begin{array}{c}\text { Total } \\
46\end{array}$} \\
\hline & \multicolumn{2}{|c|}{ G1 } & \multicolumn{2}{|c|}{ G2 } & \multicolumn{2}{|c|}{ G3 } & \multicolumn{2}{|c|}{ G4 } & \multicolumn{2}{|c|}{ G5 } & \multicolumn{2}{|c|}{ G6 } & \\
\hline & 24 & 0,52 & 0 & 0 & 9 & 0,20 & 0 & 0 & 0 & 0 & 13 & 0,283 & \\
\hline 2 & 26 & 0,54 & 1 & 0,02 & 3 & 0,06 & 0 & 0 & 0 & 0 & 18 & 0,375 & 48 \\
\hline 3 & 34 & 0,67 & 0 & 0,00 & 2 & 0,04 & 1 & 0,02 & 0 & 0 & 14 & 0,275 & 51 \\
\hline 4 & 21 & 0,34 & 2 & 0,03 & 33 & 0,54 & 0 & 0,00 & 0 & 0 & 5 & 0,082 & 61 \\
\hline 5 & 5 & 0,14 & 3 & 0,09 & 24 & 0,69 & 2 & 0,06 & 0 & 0 & 1 & 0,029 & 35 \\
\hline 6 & 12 & 0,24 & 4 & 0,08 & 26 & 0,53 & 0 & 0,00 & 0 & 0 & 7 & 0,143 & 49 \\
\hline 7 & 4 & 0,06 & 2 & 0,03 & 49 & 0,78 & 1 & 0,02 & 0 & 0 & 7 & 0,111 & 63 \\
\hline 8 & 27 & 0,55 & 0 & 0,00 & 1 & 0,02 & 0 & 0,00 & 0 & 0 & 21 & 0,429 & 49 \\
\hline 9 & 18 & 0,46 & 1 & 0,03 & 7 & 0,18 & 0 & 0,00 & 0 & 0 & 13 & 0,333 & 39 \\
\hline 10 & 15 & 0,38 & 1 & 0,03 & 5 & 0,13 & 3 & 0,08 & 0 & 0 & 15 & 0,385 & 39 \\
\hline 11 & 13 & 0,33 & 0 & 0,00 & 10 & 0,26 & 0 & 0,00 & 0 & 0 & 16 & 0,410 & 39 \\
\hline 12 & 16 & 0,34 & 5 & 0,11 & 16 & 0,34 & 0 & 0,00 & 0 & 0 & 10 & 0,213 & 47 \\
\hline 13 & 25 & 0,63 & 0 & 0,00 & 1 & 0,03 & 0 & 0 & 0 & 0 & 14 & 0,35 & 40 \\
\hline 14 & 13 & 0,37 & 0 & 0 & 12 & 0,34 & 0 & 0 & 0 & 0 & 10 & 0,286 & 35 \\
\hline 15 & 8 & 0,16 & 3 & 0,06 & 30 & 0,60 & 2 & 0,04 & 0 & 0 & 7 & 0,14 & 50 \\
\hline 16 & 21 & 0,51 & 0 & 0,00 & 5 & 0,12 & 0 & 0 & 0 & 0 & 15 & 0,366 & 41 \\
\hline 17 & 30 & 0,59 & 0 & 0,00 & 2 & 0,04 & 0 & 0 & 0 & 0 & 19 & 0,373 & 51 \\
\hline 18 & 21 & 0,51 & 1 & 0,02 & 5 & 0,12 & 0 & 0 & 0 & 0 & 14 & 0,341 & 41 \\
\hline 19 & 4 & 0,08 & 3 & 0,06 & 33 & 0,62 & 0 & 0 & 4 & 0,08 & 9 & 0,170 & 53 \\
\hline 20 & 14 & 0,19 & 0 & 0,00 & 49 & 0,66 & 0 & 0 & 1 & 0,01 & 10 & 0,135 & 74 \\
\hline 21 & 18 & 0,47 & 0 & 0,00 & 3 & 0,08 & 0 & 0 & 0 & 0,00 & 17 & 0,447 & 38 \\
\hline 22 & 37 & 0,76 & 0 & 0,00 & 1 & 0,02 & 0 & 0 & 0 & 0,00 & 11 & 0,224 & 49 \\
\hline 23 & 22 & 0,41 & 1 & 0,02 & 24 & 0,44 & 0 & 0 & 0 & 0,00 & 7 & 0,130 & 54 \\
\hline 24 & 21 & 0,40 & 3 & 0,06 & 19 & 0,36 & 0 & 0 & 1 & 0,02 & 9 & 0,170 & 53 \\
\hline 25 & 30 & 0,50 & 0 & 0 & 16 & 0,27 & 0 & 0 & 0 & 0 & 14 & 0,233 & 60 \\
\hline Total & 479 & 10,16 & 30 & 0,63 & 385 & 7,46 & 9 & 0,21 & 6 & 0,11 & 296 & 6,431 & 1205 \\
\hline Promedio & 19,2 & 0,41 & 1,20 & 0 & 15,40 & 0,30 & 0,36 & 0 & 0,24 & 0 & 11,84 & 0,257 & \\
\hline sd & 9,0 & 0,18 & 1,50 & 0 & 14,62 & 0,24 & 0,81 & 0 & 0,83 & 0 & 4,76 & 0,121 & \\
\hline
\end{tabular}

grupos presentó una diferencia significativa entre las comunidades. La diferencia en la distribución de los grupos puede ser explicada mediante la asociación con las variables $\mathrm{CE}$ y pH del suelo.
Las microfanerófitas de hojas semi-coriáceas y las nanofanerófitas de hojas suculentas se ubicaron en los extremos de un gradiente ascendente de $\mathrm{CE}$ y $\mathrm{pH}$. 
M. E. Figueroa et al. - Nanofanerófitas suculentas y salinidad

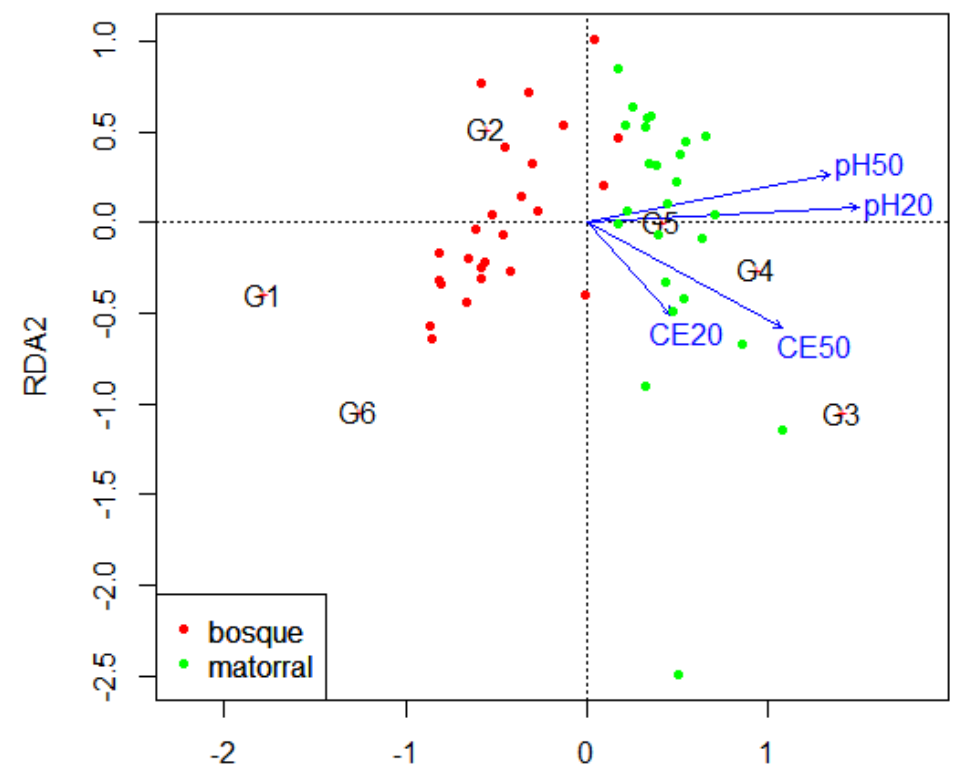

RDA1

Fig. 5. Gráfico biplot del análisis de redundancia, RDA, que relaciona las variables edáficas (CE20, CE50, $\mathrm{pH} 20, \mathrm{pH} 50$ ) y los seis grupos de plantas (G1 al G6). CE20: conductividad eléctrica de los primeros $20 \mathrm{~cm}$ de profundidad del suelo; CE50: conductividad eléctrica de 20 a $50 \mathrm{~cm}$ de profundidad del suelo; $\mathrm{pH} 20: \mathrm{pH}$ de los primeros $20 \mathrm{~cm}$ de profundidad del suelo; pH50: $\mathrm{pH}$ de 20 a $50 \mathrm{~cm}$ de profundidad del suelo. G1: microfanerófitas con hojas semi-coriáceas. G2: microfanerófitas coriáceas. G3: nanofanerófitas de hojas suculentas. G4: nanofanerófitas semi-coriáceas. G5: caméfitas suculentas. G6: especies de hoja coriácea

\section{Matorral halófito}

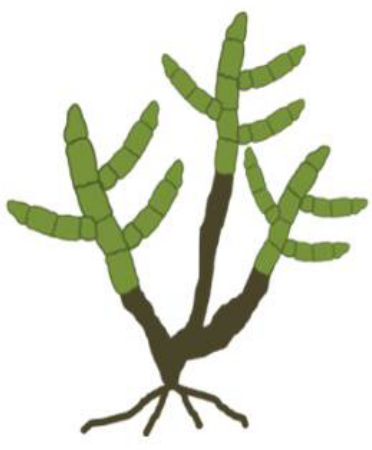

G3个

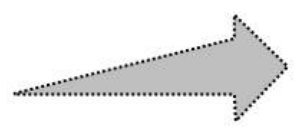

Riqueza

Diversidad

Formas de vida

\section{Bosque xerófito}

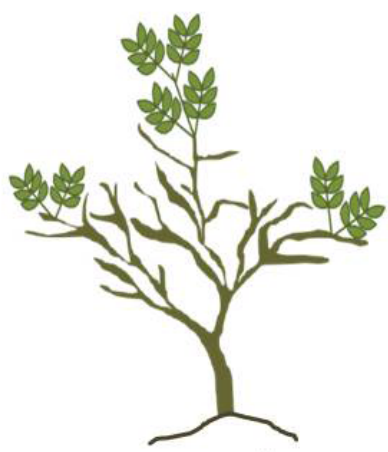

G1†

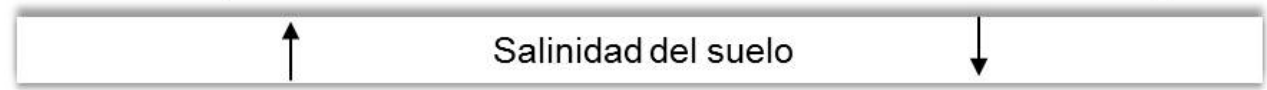

Fig. 6. Esquema de las características de las comunidades de leñosas de matorral y bosque. Grupos de plantas dominantes en cada comunidad: G3: nanofanerófitas de hojas suculentas y G1: microfanerófitas con hojas semi-coriáceas. 


\section{Discusión y Conclusiones}

En el matorral y el bosque los valores de conductividad eléctrica estuvieron por encima de los umbrales críticos para considerar un suelo salino (8 y 15 dS/m, FAO, 2006). Los cambios observados en la estructura de la vegetación entre ambos sitios se corresponden con los descriptos frecuentemente a lo largo de gradientes de salinidad (Ragonese, 1951; Díaz \& Cabido, 1997; Vendramini et al., 2002; Álvarez-Rogel et al., 2006; GonzálezAlcaraz et al., 2014). Una baja diversidad y riqueza de especies, baja cobertura vegetal y complejidad estructural se asocian a suelos con alta salinidad (Ragonese, 1951; Cabido \& Zak 1999; Martínez Carretero, 2001; Coirini et al. 2010; GonzálezAlcaraz et al., 2014; Moreno et al., 2018). Las especies relevadas son comúnmente citadas en los ambientes áridos, semiáridos y salinos de la región Chaqueña Argentina (Ragonese, 1951; Cabido \& Zak, 1999; Martínez Carretero, 2001; Vendramini et al., 2002; Taleisnik \& López Launestein, 2011). Las mismas se caracterizan por tolerar amplios rangos de salinidad (Mitlöhner, 1990; Meloni et al., 2008).

La baja medida de similitud entre ambos sitios, según el valor del coeficiente de Sørensen, estuvo relacionada con cambios en la abundancia más que con el número de especies compartidas tanto de hojas suculentas como de nanofanerófitas. El índice de Sørensen cuantitativo es empleado como una medida de diversidad beta entre dos comunidades, sustrayendo el resultado de 1; de esta manera indica el grado de cambio o reemplazo en la composición de especies entre diferentes comunidades en un paisaje (Moreno, 2001; Magurran, 2004). Así, la diversidad beta fue alta $(1-I S c u a n t=0,7)$ entre ambas comunidades leñosas del área de influencia del río Mailín, lo cual indica un alto grado de cambio en la composición de las especies de hojas suculentas y de las nanofanerófitas.

Entre las leñosas relevadas las microfanerófitas de hojas semi-coriáceas (G1) y las nanofanerófitas de hojas suculentas (G3) fueron los dos grupos más abundantes y con mayor redundancia de especies (cantidad de especies con los mismos rasgos funcionales). El grupo G1 y G3 se ubicaron en un gradiente ascendente de salinidad y alcalinidad, pudiendo así indicar diferentes estrategias de uso de recursos según el contenido de agua de la hoja. Éste es un indicador funcional en gradientes de disponibilidad hídrica donde las plantas de hojas coriáceas con bajo contenido de agua están asociadas a condiciones de estrés hídrico (Díaz \& Cabido, 1997; Vendramini et al., 2002). Es frecuente entre las especies esclerófilas con baja área foliar específica y alto espesor foliar (Vendramini et al., 2002). En cambio, las plantas de hojas herbáceas con área foliar específica alta, alto contenido de agua y bajo espesor foliar, suelen estar asociadas a ambientes con mayor disponibilidad hídrica (Vendramini et al., 2002). Sin embargo, en floras con suculentas, un mayor contenido de agua de la hoja no está asociado a una mayor disponibilidad hídrica, sino que es un rasgo respuesta a condiciones de estrés salino (Díaz \& Cabido, 1997; Vendramini et al., 2002; García et al., 2008; Pérez Cuadra \& Cambi, 2014). Por lo cual, una alta densidad de individuos con este rasgo podría indicar condiciones de alta salinidad.

Entre las especies de la región Chaqueña los tipos de hoja carnosa, sub-coriácea y coriácea son los más comunes (Arambarri et al., 2011). El desarrollo de tejidos almacenadores de agua es considerado una convergencia evolutiva entre los diferentes grupos taxonómicos de hábitats salinos (Vendramini et al., 2002; Eggli \& Nyffeler, 2009; Pérez Cuadra \& Cambi, 2014; Conti et al., 2017). Las nueve especies que compartieron el grupo G3 de las nanofanerófitas suculentas están distribuidas en cinco familias, entre las cuales las especies del género Lycium (Solanaceae) fueron predominantes (Bernardello, 2013). Díaz \& Cabido (1997) identificaron un tipo funcional de planta (TFP7) para la flora del centro-oeste de Argentina, que predomina en la vegetación halófita y comparte características semejantes a las del grupo G3, aunque con un menor número de especies $(4 \mathrm{sp})$. Conti et al. (2017) encontraron que las especies funcionalmente similares, en términos de uso del recurso hídrico, convergen en micro-parches con igual salinidad. Es decir, que las leñosas de hojas suculentas podrían estar ocupando nichos de mayor salinidad en escalas espaciales finas dentro de cada comunidad.

A pesar de que puede existir una fuerte asociación taxonómica entre las especies de un grupo funcional, la relevancia ecológica de los mismos no se modifica (Díaz \& Cabido, 1997). En el G1 fueron dominantes especies de porte arbustivo, arbóreo y de hojas semi-coriáceas, de la familia Fabaceae, con 
cinco especies típicas de la región chaqueña. Las tres especies del G4, más abundantes en el matorral, al igual que las del G1 poseen hojas semi-coriáceas, aunque se diferencian en la forma de vida arbustiva $\mathrm{y}$ en que presentan adaptaciones a la salinidad. Entre ellas, Ximenia americana (Olacaceae), es descripta como especie de ambiente cálido, soleado y tolerante a la salinidad (Arambarri et al., 2011); Prosopis reptans, es un arbusto espinoso endémico de Argentina, con órganos subterráneos invasores de suelos salinos y Prosopis sericantha es un arbusto subáfilo espinoso de las regiones argentinas Chaqueña Occidental y Monte (Ragonese, 1951; Flora Argentina, 2017). Las tres especies del G2, Maytenus spinosa, Prosopis vinalillo y Prosopis ruscifolia poseen hojas coriáceas y características esclerófilas, con adaptaciones a la salinidad. Sin embargo, estuvieron escasamente representadas en ambos sitios, a pesar de que podría esperarse una alta densidad de individuos de Prosopis ruscifolia ya que es la especie arbórea colonizadora típica de estos ambientes (Meloni et al., 2008).

En el G5, Alternanthera nodifera y Ehretia cortesia son especies halófitas de hojas suculentas importante forrajeras (Gottschling \& Hilger, 2004; Cavanna et al., 2010), estuvieron escasamente representadas en ambos sitios. En el grupo G6, las cinco especies pertenecen a familias distintas, dos son especies arbóreas del dosel del bosque Chaqueño, Schinopsis lorentzii y Aspidosperma quebracho-blanco; Atamisquea emarginata y Condalia microphylla son arbustos típicos del bosque xerófito. Estas cuatro especies presentan hojas esclerófilas, baja área foliar específica y bajo contenido de agua en la hoja, los cuales son rasgos funcionales de respuesta al estrés hídrico (Díaz \& Cabido, 1997; Vendramini et al., 2002). A excepción de dichas especies, que fueron más abundantes en el bosque, Lippia salsa, caméfita de hoja coriácea, es una halófita forrajera (Cavanna et al., 2010), que fue incluida en el G6 por compartir el rasgo de hoja coriácea, pero fue más abundante en el matorral.

Otros autores han propuesto modelos conceptuales para ambientes salinos basados en la vegetación como bioindicador de las condiciones edáficas (Álvarez-Rogel et al., 2006; GonzálezAlcaraz et al., 2014; Moreno et al., 2018). Generalmente las suculentas indican salinidades extremas y marcan zonas de vegetación suculenta versus no suculenta (Moreno et al., 2018). Estas especies suelen ser las primeras colonizadoras bajo la más alta salinidad del suelo (ÁlvarezRogel et al., 2006). González-Alcaraz et al. (2014) encontraron que las suculentas se convierten en dominantes con incrementos de la CE por encima de los $52 \pm 20 \mathrm{dS} / \mathrm{m}$. Los resultados del modelo propuesto en el DRA permitirían reconocer un tipo funcional de respuesta a condiciones de salinidad conformado por leñosas nanofanerófitas de hojas suculentas. Sin embargo sería necesario incrementar los muestreos y ampliar el área de estudio para mejorar la capacidad de extrapolación de los resultados y la aplicabilidad de las relaciones encontradas en comunidades leñosas de ambientes salinos semiáridos.

\section{ConTribución DE LOS AUTORES}

MEF realizó la investigación, análisis, recolección e interpretación de datos, preparación de las figuras, redacción del manuscrito. JGM realizó la conceptualización de la investigación. GL realizó el diseño de muestreo, recolección de muestras, análisis en laboratorio y descripción taxonómica del suelo. AMG gestionó y coordinó la planificación y ejecución de la investigación. Todos los autores participaron en la interpretación de los resultados y redacción del manuscrito.

\section{Bibliografía}

ÁLVAREZ-ROGEL, J., J. J. MARTÍNEZ-SÁNCHEZ, L. C. BLÁZQUEZ \& C. M. M. SEMITIEL. 2006. A conceptual model of salt marsh plant distribution in coastal dunes of southeastern Spain. Wetlands 26: 703-717.

https://doi.org/10.1672/0277. 5212(2006)26[703:ACMOSM]2.0.CO;2

ARAMBARRI, A. M., M. C. NOVOA, N. D. BAYON, M. P. HERNÁNDEZ, M. N. COLARES \& C. MONTI. 2011. Ecoanatomía foliar de árboles y arbustos de los distritos chaqueños occidental y serrano (Argentina). Bol. Soc. Argent. Bot. 46: 251270.

ARAMBARRI, A., C. MONTI, N. BAYÓN, M. HERNÁNDEZ, M. C. NOVOA \& M. COLARES. 2012. Ecoanatomía foliar de arbustos y árboles del distrito chaqueño oriental de la Argentina. Bonplandia 21: 5-26. 
ASTRADA, E. \& J. ADÁMOLI. 2005. Ecología y manejo de vinalares. Perspectiva regional y aplicaciones en el centro de Formosa. En: ARTURI, M. F., J. L. FRANGI \& J.F. GOYA (eds.) Ecología y manejo de bosques de Argentina. Editorial de la Universidad Nacional de La Plata, Buenos Aires.

ATIA, A., M. RABHI, A. DEBEZ, C. ABDELLY, H. GOUIA, C. C. HAOUARI \& A. SMAOUI. 2014. Ecophysiological aspects and photosynthetic pathways in 105 plants species in saline and arid environments of Tunisia. J. Arid Land 6: 762-770. https://doi.org/10.1007/s40333-014-0028-2

BERNARDELLO, G. 2013. Lycium. En: BARBOZA, G. (ed.) Flora Argentina, Dycotiledoneae, Solanaceae, pp. 47-75. INTA, IMBIV, IBODA, Buenos Aires.

BERTILLER, M. B., A. J. BISIGATO, A. L. CARRERA \& H. F. DEL VALLE. 2004. Estructura de la vegetación y funcionamiento de los ecosistemas del Monte Chubutense. Bol. Soc. Argent. Bot. 39: 139-158.

BRAUN -BLANQUET, J. 1979. Fitosociología. Bases para el estudio de las comunidades vegetales. Ed. H. Blume, Madrid.

BUI, E.N. 2013. Soil salinity: A neglected factor in plant ecology and biogeography. J. Arid Environm. 92: 14-25. https://doi.org/10.1016/j.jaridenv.2012.12.014

CABIDO, M. \& M. ZAK. 1999. La vegetación del norte de Córdoba. Secretaría de Agricultura, Ganadería y Rec. Renov. Córdoba, Córdoba, Argentina.

CABRERA, A. 1976. Regiones fitogeográficas argentinas. Fascículo 1. Enciclopedia Argentina de Agricultura y Jardinería. Segunda Edición, Tomo II. Editorial ACME SACI

CAVANNA, J., G. CASTRO, U. KARLIN \& M. KARLIN. 2010. Ciclo ganadero y especies forrajeras en Salinas Grandes, Catamarca, Argentina. Zonas Áridas 14: 170-180.

CHAPIN, F.S. 1993. Functional role of growth forms in ecosystem and global processes. In: EHLERINGER, J.R. \& C.B. FIELD (eds.) Scaling physiological processes: leaf to globe, pp. 287-312. Academic Press, San Diego, CA. https://doi.org/10.1016/B978-0-12-233440-5.500245

CONTI, L., F. DE BELLO, J. LEPŠ, A.T.R. ACOSTA \& M. CARBONI. 2017. Environmental gradients and micro-heterogeneity shape fine-scale plant community assembly on coastal dunes. J. Veg. Sci. 28: 762-773.

https://doi.org/10.1111/jvs.12533

DI RIENZO, J.A., F. CASANOVES, M.G BALZARINI, L. GONZALEZ, M. TABLADA \& C. W. ROBLEDO. 2014. InfoStat versión 2014. Grupo InfoStat, FCA, Universidad Nacional de Córdoba, Argentina.
DÍAZ, S. \& M. CABIDO. 1997. Plant functional types and ecosystem function in relation to global change. $J$. Veg. Sci. 8: 463-474. https://doi.org/10.1111/j.1654-1103.1997.tb00842.x

EGGLI, U. \& R. NYFFELER. 2009. Living under temporarily arid conditions-succulence as an adaptive strategy. Bradleya 27: 13-36. https://doi.org/10.25223/brad.n27.2009.a10

FAO 2006. World reference base for soil resources 2006. A framework for international classification, correlation and communication. World Soil Resources Reports 103. Rome.

FLORA ARGENTINA. 2017. Flora vascular de la República Argentina [online]. Disponible en: http:// www.floraargentina.edu.ar/ [Acceso: 27 junio 2017].

GARCÍA, M., D. JÁUREGUI \& E. MEDINA. 2008. Adaptaciones anatómicas foliares en especies de angiospermas que crecen en la zona costera del estado Falcón (Venezuela). Acta Bot. Venez. 31: 291-306.

GIMÉNEZ, A. M. \& J. G. MOGLIA. 2003. Árboles del Chaco Argentino: guía para el reconocimiento dendrológico. Universidad Nacional de Santiago del Estero.

GIMÉNEZ, A. M., M. E. FIGUEROA, P. HERNÁNDEZ \& M. CEJAS. 2008. Jume negro (Allenrolfea vaginata Kuntze, Chenopodiaceae): potencialidades de usos en la rigurosidad del ambiente salino. Quebracho 15: 32-36.

GIMÉNEZ, A. M., N. RÍOS, P. HERNÁNDEZ \& J. G. MOGLIA. 2009. Influencia de la edad en el crecimiento de vinal (Prosopis ruscifolia Burkart.), en la Provincia de Santiago del Estero, Argentina. Madera y bosques 15: 45-57. https://doi.org/10.21829/myb.2009.1521190

GONZÁLEZ-ALCARAZ, M. N., F. J. JIMÉNEZCÁRCELES, Y. ÁLVAREZ \& J. ÁLVAREZ-ROGEL. 2014. Gradients of soil salinity and moisture, and plant distribution, in a Mediterranean semiarid saline watershed: a model of soil-plant relationships for contributing to the management. Catena 115: 150-158. https://doi.org/10.1016/j.catena.2013.11.011

GOTTSCHLING, M. \& H. H. HILGER. 2004. The systematic position of Ehretia cortesia nom. nov.(三 Cortesia cuneifolia: Ehretiaceae, Boraginales) inferred from molecular and morphological data. Taxon 53: 919-923. https://doi.org/10.2307/4135559

GRIGORE, M.N., C. TOMA \& M. BOŞCAIU. 2010. Dealing with halophytes: an old problem, the same continuous exciting challenge. Analele știinţifice ale Universităţii “Al. I. Cuza” Iaşi, Biologie vegetală , Tomul LVI: s.II a.

HUCHZERMEYER, B. A. \& T. FLOWERS. 2013. Putting halophytes to work-genetics, biochemistry and physiology. Functional Plant Biology 40: v-viii. https://doi.org/10.1071/FPv40n9 FO 


\section{E. Figueroa et al. - Nanofanerófitas suculentas y salinidad}

IUSS Working Group WRB 2014. World Reference Base for Soil Resources 2014. International soil classification system for naming soils and creating legends for soil maps. World Soil Resources Reports 106. FAO Rome.

KOYRO, H. W., N. GEISSLER, S. HUSSIN \& B. HUCHZERMEYER. 2008. Mechanisms of cash crop halophytes to maintain yields and reclaim saline soils in arid areas. In: KHAN, M.A. \& D.J. WEBER (eds.) Ecophysiology of high salinity tolerant plants, pp. 345366. Springer, Netherlands. https://doi.org/10.1007/1-4020-4018-0_22

LARCHER, W. 1977. Ecofisiología Vegetal. Ediciones Omega.

MAGURRAN, A. 2004. Measuring Biological Diversity. Oxford, RU: Blackwell Publishing.

MARGALEF, R. 1974. Ecología. Omega. Barcelona.

MARTÍNEZ CARRETERO, E. 2001. Esquema sintaxonómico de la vegetación de regiones salinas de Argentina. Multequina 10: 67-74.

MELONI, D. A., M. R. GULOTTA \& C. A. MARTÍNEZ. 2008. Prosopis ruscifolia Griseb. (vinal) tolera concentraciones salinas equivalentes al agua de mar y excluye iones tóxicos de la parte aérea. Quebracho 16: $32-40$.

MILLER, J. 1989. A revision of the New World species of Ehretia (Boraginaceae). Ann. Missouri Bot. Gard. 76: 1050-1076. https://doi.org/10.2307/2399691

MITLÖHNER, R. 1990. Competition of woody plants in the rainy green dry forest of Chaco Boreal, Paraguay [Die Konkurrenz der Holzgewächse im regengrünen Trockenwald des Chaco Boreal, Paraguay. Universität Göttingen, Göttinger Beitr. Zur Land- und Forstwirtschaft in den Tropen und Subtropen], E. Goltze, Germany.

MORENO, C.E. 2001. Métodos paramedir la biodiversidad. M\&T-Manuales y Tesis SEA, vol.1. Zaragoza.

MORENO, J., A. TERRONES, A. JUAN \& M. Á. ALONSO. 2018. Halophytic plant community patterns in Mediterranean saltmarshes: shedding light on the connection between abiotic factors and the distribution of halophytes. Plant and Soil 430: 185-204.

https://doi.org/10.1007/s11104-018-3671-0

MOSCATELLI, G. 1990. Atlas de suelos de la República Argentina. Proyecto PNUD, Argentina 85-019, Secretaría de Agricultura y Ganadería de la Nación, Instituto Nacional de Tecnología Agropecuaria, Buenos Aires.

NOURI, H., S. C. BORUJENI, R. NIROLA, A. HASSANLI, S. BEECHAM, S ALAGHMAND... \& D. MULCAHY. 2017. Application of green remediation on soil salinity treatment: a review on halophytoremediation. Process Safety and Environmental Protection 107: 94-107. https://doi.org/10.1016/j.psep.2017.01.021
OKSANEN, J., F. G. BLANCHET, M. FRIENDLY, R. KINDT, P. LEGENDRE, D. MCGLINN, P. R. MINCHIN, R. B. O'HARA, G. L. SIMPSON, P. SOLYMOS, M. HENRY, H. STEVENS, E. SZOECS \& H. WAGNER. 2018. Vegan: Community Ecology Package. R package version 2.5-2. Disponible en: https://cran.ism.ac.jp/web/packages/vegan/vegan. pdf [Acceso: 1 Agosto 2018].

PANTA, S., T. FLOWERS, P. LANE, R. DOYLE, G. HAROS \& S. SHABALA. 2014. Halophyte agriculture: success stories. Environm. Exp. Bot. 107: 71-83. https://doi.org/10.1016/j.envexpbot.2014.05.006

PÉREZ CUADRA, V. \& V. N. CAMBI. 2014. Ocurrencia de caracteres anatómicos funcionales foliares y caulinares en 35 especies xero-halófilas. Bol. Soc. Argent. Bot. 49: 347-359.

PIERNIK, A. 2003. Inland halophilous vegetation as indicator of soil salinity. Basic and Applied Ecology 4: 525-536. https://doi.org/10.1078/1439-1791-00154

PRIETO, D., A. BIANCHI, L. ACUÑA, R. NIEVA, R. PEDRAZA, G. ANGELlA, H. ELENA, H. VAN MEER \& F. TOLABA. 2006. Sistema de Procesamiento de Datos Agrometereológicos del NOA (ProDaAgro v.1.0) INTA EEA Santiago del Estero. En: ANGUEIRA, C., D. PRIETO, L. LÓPEZ \& G. BARRAZA (2007) Sistema de Información Geográfica de Santiago del Estero (SigSE 2.0) CD Rom. INTA EEA Santiago del Estero.

R DEVELOPMENT CORE TEAM. 2015. R: A language and environment for statistical computing. R Foundation for Statistical Computing, Vienna, Austria. ISBN 3-900051-07-0, Disponible en: http:// www.R-project.org.

RAGONESE, A. \& J. CASTIGLIONE. 1970. La vegetación del Parque Chaqueño. Bol. Soc. Arg. Bot. 11: 133-160.

RAGONESE, A. 1951. La vegetación de la República Argentina. II.- Estudio fitosociológico de las Salinas Grandes. Rev. Inv. Agr. 51:1-233.

RÍOS, N., M. CEJAS \& M. MALDONADO. 2008. El vinal (Prosopis ruscifolia Grises.) una especie importante en el Gran Chaco Americano, Argentina. Foresta Veracruzana 10:17-26.

RUAN, C. J., J. A. T. DA SILVA, S. MOPPER, P. QIN \& S. LUTTS. 2010. Halophyte improvement for a salinized world. Crit. Rev. Pl. Sci. 29: 329-359. https://doi.org/10.1080/07352689.2010.524517

SCHLESINGER, W. H., J. A. RAIKES, A. E. HARTLEY \& A. F. CROSS. 1996. On the spatial pattern of soil nutrients in desert ecosystems. Ecology 77:364-374. https://doi.org/10.2307/2265615

SCHOFIELD, R. V. \& M. J. KIRKBY. 2003. Application of salinization indicators and initial development of potential global soil salinization scenario under climatic change. Global Biogeochem. Cycles 17: 1078. https://doi.org/10.1029/2002GB001935 
SOIL SURVEY STAFF. 2014. Keys to Soil Taxonomy. USDA-Natural Resources Conservation Service, Washington, DC, 12 edition.

SUBSECRETARÍA DE RECURSOS HÍDRICOS DE LA NACIÓN ARGENTINA, 1979. Programa para el estudio integral Río Dulce. Subprograma geomorfología y evaluación de escurrimientos. Informe final Santiago del Estero- Córdoba.

TALEISNIK, E. \& D. LÓPEZ LAUNESTEIN. 2011. Leñosas perennes para ambientes afectados por salinidad. Una sinopsis de la contribución argentina a este tema. Ecol. Austral 21: 3-14.

THE PLANT LIST, 2013. Version 1.1 [online]. Disponible en: http://www.theplantlist.org/ [Acceso: 1 Agosto 2018].

TÜRKAN， I. \& T. DEMIRAL. 2009. Recent developments in understanding salinity tolerance. Environm. Exp. Bot. 67: 2-9. https://doi.org/10.1016/j.envexpbot.2009.05.008

VENDRAMINI, F., S. DÍAZ, D. E. GURVICH, P. J. WILSON, K. THOMPSON \& J. G. HODGSON 2002. Leaf traits as indicators of resource-use strategy in floras with succulent species. New Phytol. 154: $147-157$. https://doi.org/10.1046/j.1469-8137.2002.00357.x WILDER, B. T., R. S. FELGER \& H. R. MORALES. 2008. Succulent plant diversity of the Sonoran islands, Gulf of California, Mexico. Haseltonia 127-160. https://doi.org/10.2985/1070-0048-14.1.127

YANG, F., G. ZHANG, X. YIN \& Z. LIU. 2011. Fieldscale spatial variation of saline-sodic soil and its relation with environmental factors in Western Songnen Plain of China. Int. J. Environ. Res. Public Health 8: 374-387.

https://doi.org/10.3390/ijerph8020374 